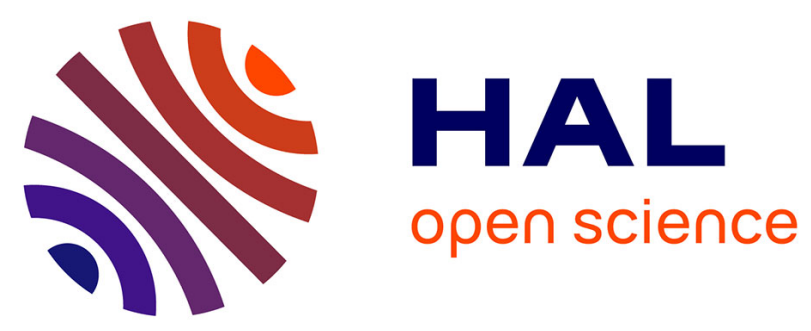

\title{
Optimal Sensor Placement for Partially Known Power System Dynamic Estimation
}

\author{
Mohammad Ali Abooshahab, Morten Hovd, Giorgio Valmorbida
}

\section{To cite this version:}

Mohammad Ali Abooshahab, Morten Hovd, Giorgio Valmorbida. Optimal Sensor Placement for Partially Known Power System Dynamic Estimation. IEEE PES ISGT Europe 2021, Oct 2021, Espoo, Finland. hal-03488243

\section{HAL Id: hal-03488243 https://hal.science/hal-03488243}

Submitted on 17 Dec 2021

HAL is a multi-disciplinary open access archive for the deposit and dissemination of scientific research documents, whether they are published or not. The documents may come from teaching and research institutions in France or abroad, or from public or private research centers.
L'archive ouverte pluridisciplinaire HAL, est destinée au dépôt et à la diffusion de documents scientifiques de niveau recherche, publiés ou non, émanant des établissements d'enseignement et de recherche français ou étrangers, des laboratoires publics ou privés. 


\title{
Optimal Sensor Placement for Partially Known Power System Dynamic Estimation
}

\author{
Mohammad Ali Abooshahab, Morten Hovd, and Giorgio Valmorbida
}

\begin{abstract}
The synchronized phasor measurement unit (PMU) provides fast, precise, and synchronized measurements, which is crucial for power systems dynamic monitoring. However, replacing conventional SCADA systems with PMUs is happening gradually and slowly due to its considerable cost. Hence, with restricted budgets, the installation of these measuring devices should be selective. The majority of previous works on PMU sensor placement have been focused on steady-state estimation. Therefore, they concentrate on network topology to find optimal configurations making the whole network observable at steady-state. However, approaches based on steady-state or quasi-steady-state operating conditions are not applicable for power systems experiencing fast and dynamic changes. Moreover, a dynamic model for several variables and parts in a power system may be wholly or partly unknown. In such cases, the concept of partially known power systems as well as simultaneous input and state estimation can be exploited to obtain the estimates of the known system states and unknown inputs. In this work, we develop a greedy approach to obtain optimal sensor placement for joint input and state estimation of partially known power grids.
\end{abstract}

\section{INTRODUCTION}

Monitoring is significantly essential for modern electrical grids' energy management systems. Thanks to the introduction and development of PMUs, more frequent measurements are accessible in different parts of a power grid. This allows for the exploitation of dynamic approaches for power grid monitoring instead of steady-state or quasi-steady-state estimation. In this case, it is possible to track sudden changes, including electromagnetic transients [1], cyber-attacks [2], [3], and dynamics of distributed generators [4]. However, in practice, few nodes can be covered by PMUs because they are expensive devices. Thus, it is necessary to place the PMUs selectively and optimally. Several methods have been conducted on PMU placement problems. A summary of different approaches to dealing with the sensor placement problem can be found in [5]. One of the most popular methods to address the sensor placement optimization problem is the greedy algorithm [6], [7] due to its simplicity.

There are several interconnected elements or layers in an electrical grid, such as distribution, transmission, generation,

\footnotetext{
Mohammad Ali Abooshahab and Morten Hovd are with the Department of Engineering Cybernetics, Norwegian University of Science and Technology, Trondheim, Norway, (e-mails: \{mohammad.ali.abooshahab, morten. Hovd\} (antnu.no).

Giorgio Valmorbida is with the CentraleSupelec, University of Paris Plateau de Moulon, 3 rue Joliot-Curie, Gif-sur-Yvette Cedex, France. (email: giorgio.valmorbida@l2s.centralesupelec.fr)

This work is funded by CINELDI - Centre for intelligent electricity distribution, an 8-year Research Centre under the FME-scheme (Centre for Environment-friendly Energy Research, 257626/E20). The authors gratefully acknowledge the Research Council of Norway and the CINELDI partners' financial support.
}

and loads. Many parts of the system may be fully known with the information required for dynamic modeling fully available, while other regions may be entirely or partially unknown. Moreover, the availability of the measurements in some parts of the modern electrical grids, especially in the distribution part, is limited. This is due to the consumers' privacy and the lack of expensive and accurate measuring devices such as PMUs. Hence, the partially known power system concept has been recently introduced in [8]. In this context, the Kalman Filtering - Simultaneous Input and State Estimation (KF-SISE), proposed and investigated in [9], [10], is utilized to obtain the estimates of the states and unknown inputs of the partially known power grid. It should be borne in mind that various sensor placement methods based on steady-state/quasi-steady-state conditions, including those proposed in [11], [12], [13] are not applicable for modern electrical grids facing fast and dynamic changes. In addition, these approaches require more sensors than the total number of states in the system, which means they usually need more measurements than their dynamic counterparts [2]. Similarly, sensor placement strategies ensuring observability of the overall system [14], [15], [16], [17] require an excessive number of sensors. The proposed method in this paper, however, requires fewer measurements in an extensive power network. It requires observability of the known part of the system, and in addition only one measurement per signal originating in the unknown parts of the system.

The aim of this paper is, first, to formulate the optimization problem to solve optimal sensor placement for dynamic state estimation of partially known systems. To achieve this goal, we obtain the information matrix corresponding to KFSISE and then propose 'best in' and 'worst out' greedy algorithms to optimize the placement of sensors in a power network. The criteria for the minimization in such problems is usually the covariance of estimation error. However, estimation error for the unknown input is also critical for obtaining the overall uncertainty originated using KF-SISE. Hence, we introduce a new objective function consisting of both the covariance of estimation error and unknown input uncertainty. Next, by proving that adding a new measurement does not corrupt the estimation performance of KF-SISE, we simplify the inequality constraint of the optimization problem into equality. Finally, we present the results of numerical simulations on the Western Systems Coordinating Council (WSCC) 3-machine-9-bus test system and the IEEE-14-bus test system. The results are compared with approaches in the literature and validate the performance of the proposed sensor placement method for KF-SISE based state estimation of partially known power networks. 
The structure of this paper is as follows. Section II introduces KF-SISE and the concept of partially known power systems. In Section III, we formulate the sensor placement problem, and we solve this optimization problem using the greedy algorithm. We present the simulation results related to the WSCC-9-bus and IEEE-14-bus network in Section IV to show the performance of the proposed sensor selection method.

\section{PARTIALly KNOWN POWER NETWORKS STATE ESTIMATION}

We aim to minimize the overall covariance of the estimation error for the same number of PMUs. We first describe the filtering method. A summary of KF-SISE, presented in [9], is given in the following subsection.

\section{A. Filtering algorithm}

To simplify matters, we consider initially a linear timeinvariant formulation without direct feedthrough for the known system:

$$
\begin{aligned}
x_{t+1} & =A x_{t}+G d_{t}+w_{t}, \\
y_{t} & =C x_{t}+v_{t},
\end{aligned}
$$

where $x_{t}, w_{t} \in \mathbb{R}^{n}$ are the state vector and process noise at time step $t, d_{t} \in \mathbb{R}^{m}$ is the disturbance signal from the unknown part of the system, and $y_{t}, v_{t} \in \mathbb{R}^{m}$ are the measurements and the measurement noise. The following assumptions are required to use the Kalman filtering formulation of SISE [9].

Assumption 1: The following is assumed to hold:

i $w_{t} \sim \mathcal{N}(0, Q), v_{t} \sim \mathcal{N}(0, R)$ and initial condition $x_{O} \sim$ $\mathcal{N}\left(\hat{x}_{0 \mid 0}, P_{O}\right)$ are mutually independent Gaussian white noises,

ii $R$ is diagonal positive definite matrix,

iii the pair $(A, C)$ is observable, and $\operatorname{rank} C G=\operatorname{rank} G=$ $m$.

By defining $\mathcal{X}_{t+1}, \mathcal{K}_{t+1}, \mathcal{M}_{t+1}, \mathcal{D}_{t}$ and $\mathcal{P}_{t+1}$ respectively as the prior state covariance matrix, the Kalman gain for the state vector, the Kalman gain for the unknown input vector, the posterior disturbance covariance matrix and the posterior state covariance matrix, and $\hat{x}_{t+1 \mid t+1}$ as the posterior estimates for the state and $\hat{d}_{t \mid t+1}$ as the estimate for the unknown input of the system, and the measurement sequence, $\mathbf{Y}^{t+1} \triangleq\left\{y_{t+1}, y_{t}, \ldots, y_{1}\right\}$, we state an algorithm for KF-SISE in Algorithm 1.

\section{B. Information matrix derivation for KF-SISE}

In this subsection, we derive the information matrix for KF-SISE [9], which can simplify the analysis of estimation error and its covariance further in this study. The following lemma provides us with the information version of the KFSISE.

Lemma 1: Considering (3)-(8), the Information matrix $\mathfrak{I}_{t+1} \triangleq \mathcal{P}_{t+1}^{-1}$ for the SISE can be obtained as follows:

$$
\Im_{t+1}=\mathcal{X}_{t+1}^{-1}+C^{T} R^{-1} C
$$

Proof: Let us show that $\mathfrak{I}_{t+1}=\mathcal{P}_{t+1}^{-1}$. This will be done by showing that $\mathfrak{I}_{t+1} \mathcal{P}_{t+1}=I$, using the expression for $\mathcal{P}_{t+1}$ in (8).
Algorithm 1 State and disturbance estimation using KFSISE

For positive definite matrix $D$ large,

Prediction step: compute $\mathcal{X}_{t+1}, \mathcal{K}_{t+1}, \mathcal{M}_{t+1}$ as follows:

$$
\begin{aligned}
\mathcal{X}_{t+1} & =A \mathcal{P}_{t} A^{T}+G D G^{T}+Q \\
\mathcal{K}_{t+1} & =\mathcal{X}_{t+1} C^{T}\left(C \mathcal{X}_{t+1} C^{T}+R\right)^{-1} \\
\mathcal{M}_{t+1} & =D G^{T} C^{T}\left(C \mathcal{X}_{t+1} C^{T}+R\right)^{-1}
\end{aligned}
$$

Update step: compute $x_{t+1 \mid t+1}, d_{t \mid t+1}, P_{t+1}$ and $D_{t}$, as follows:

$$
\begin{aligned}
\hat{x}_{t+1 \mid t+1} & =A \hat{x}_{t \mid t}+G \mathfrak{d}+\mathcal{K}_{t+1}\left(y_{t+1}-C A \hat{x}_{t \mid t}-C G \mathfrak{d}\right), \\
\hat{d}_{t \mid t+1} & =\mathfrak{d}+\mathcal{M}_{t+1}\left(y_{t+1}-C A \hat{x}_{t \mid t}-C G \mathfrak{d}\right) \\
\mathcal{P}_{t+1} & =\left(I-\mathcal{K}_{t+1} C\right) \mathcal{X}_{t+1} \\
\mathcal{D}_{t} & =\left(I-\mathcal{M}_{t+1} C G\right) D
\end{aligned}
$$

Note that $\mathcal{P}_{t+1}^{-1}$ is full rank due to the observability condition in Assumption 1. We have, using (4):

$$
\begin{aligned}
\mathfrak{I}_{t+1} \mathcal{P}_{t+1} & =\left(\mathcal{X}_{t+1}^{-1}+C^{T} R^{-1} C\right)\left(I-K_{t+1} C\right) \mathcal{X}_{t+1} \\
& =\left(\mathcal{X}_{t+1}^{-1}+C^{T} R^{-1} C\right) \\
& \times\left(\mathcal{X}_{t+1}-\mathcal{X}_{t+1} C^{T}\left(C \mathcal{X}_{t+1} C^{T}+R\right)^{-1} C \mathcal{X}_{t+1}\right) \\
& =I-C^{T}\left[\left(C \mathcal{X}_{t+1} C^{T}+R\right)^{-1}-R^{-1}\right. \\
& \left.+R^{-1} C \mathcal{X}_{t+1} C^{T}\left(C \mathcal{X}_{t+1} C^{T}+R\right)^{-1}\right] C \mathcal{X}_{t+1} \\
& =I-C^{T}\left[\left(C \mathcal{X}_{t+1} C^{T}+R\right)^{-1}\left(I+R^{-1} C \mathcal{X}_{t+1} C^{T}\right)\right. \\
& \left.-R^{-1}\right] \times C \mathcal{X}_{t+1} \\
& =I-C^{T}\left[R^{-1}-R^{-1}\right] C \mathcal{X}_{t+1} \\
& =I
\end{aligned}
$$

Since the inverse of a full rank matrix is unique, we conclude $\mathfrak{I}_{t+1}=\mathcal{P}_{t+1}^{-1}$.

\section{Steady state formulation of estimation}

For the steady-state Kalman filter version of SISE, KFSISE, we would have to solve an ARE (11) to obtain $\mathcal{X}_{\infty}$ and then (12)-(15) follow [9]:

$$
\begin{aligned}
\mathcal{X}_{\infty} & =\operatorname{dare}\left(A^{T}, C^{T}, Q+G D G^{T}, R\right) \\
\mathcal{K}_{\infty} & =\mathcal{X}_{\infty} C^{T}\left(C \mathcal{X}_{\infty} C^{T}+R\right)^{-1} \\
\mathcal{M}_{\infty} & =D G^{T} C^{T}\left(C \mathcal{X}_{\infty} C^{T}+R\right)^{-1} \\
\mathcal{P}_{\infty} & =\left(I_{n}-C \mathcal{K}_{\infty}\right) \mathcal{X}_{\infty} ; \\
\mathcal{D}_{\infty} & =\left(I_{m}-\mathcal{M}_{\infty} C G\right) D
\end{aligned}
$$

The ARE solution here denoted $\mathcal{X}_{\infty}$, is the steady-state prediction error covariance from (3). $\mathcal{P}_{\infty}$ indicates the expected state estimation error corresponding to the given measurement matrix. For the same number of PMUs, we aim to minimize this overall error.

\section{Model for the test system}

This subsection is devoted to describe a dynamic model for the power network and to clarify the partially known power network concept. To derive a dynamic model for our power network, the following assumptions are considered [8]: 


\begin{tabular}{|l|l|l|l|l|l|}
\hline \multicolumn{2}{|c|}{ State variables } & \multicolumn{2}{c|}{ External variables } & \multicolumn{2}{c|}{ Parameters } \\
\hline angular frequency & $\omega_{r}$ & external load & $P_{G}$ & rotor damping coeficient & $\mathrm{D}$ \\
\hline mechanical power & $P_{m}$ & frequency setpoint & $f_{O}$ & time constant & $T_{S}$ \\
\hline rotor shaft angle & $\delta$ & load setpoint & $L_{c r e}$ & governor feedback gain & $k$ \\
\hline valve position & $a$ & & & droop characteristic & $R$ \\
\hline & & & & motor inertia & $M$ \\
\hline
\end{tabular}

TABLE I: Variables and parameters for components in the test cases.

Assumption 2: The following is assumed to hold:

i Transformers are neglected because their impedances are negligible compared to the impedances of the transmission lines.

ii The ratio of the transmission/distribution lines reactance to their resistance is assumed to be significantly above unity [18].

iii The power angle at Bus 1 is assumed to be the network's reference power angle.

In the test systems, synchronous generators and loads contribute to the power system dynamics. Generators dynamic model can be described as [19], [20]:

$$
\begin{aligned}
\frac{\partial}{\partial t}\left[\begin{array}{c}
\omega_{r} \\
P_{m} \\
a \\
\delta
\end{array}\right]_{k} & {\left[\begin{array}{cccc}
-D / M & -1 / M & 0 & 0 \\
0 & -1 / T_{S} & 1 / T_{S} & 0 \\
k & 0 & -k R & 0 \\
1 & 0 & 0 & 0
\end{array}\right]_{k}\left[\begin{array}{c}
\omega_{r} \\
P_{m} \\
a \\
\delta
\end{array}\right]_{k} } \\
& +\left[\begin{array}{cc}
0 & 0 \\
0 & 0 \\
-k & -k \\
0 & 0
\end{array}\right]_{k}\left[\begin{array}{c}
L_{c r e} \\
\omega_{O}
\end{array}\right]_{k}+\left[\begin{array}{c}
-1 / M \\
0 \\
0 \\
0
\end{array}\right]_{k} P_{G k},
\end{aligned}
$$

where variables and parameters are given in Table I. Similarly, buses containing rotating loads with known $P_{L}$ is:

$$
\begin{aligned}
\frac{\partial}{\partial t}\left[\begin{array}{c}
\omega_{r} \\
\delta
\end{array}\right]_{k}=\left[\begin{array}{cc}
-D / M & 0 \\
1 & 0
\end{array}\right]_{k}\left[\begin{array}{c}
\omega_{r} \\
\delta
\end{array}\right]_{k} \\
+\left[\begin{array}{c}
-1 / M \\
0
\end{array}\right]_{k} P_{L k}+\left[\begin{array}{c}
-1 / M \\
0
\end{array}\right]_{k} P_{G k} .
\end{aligned}
$$

The model derivation process for the WSCC-9-bus network as in [8] is given in the Appendix. Aggregating all states of different components (machines, loads, condensers, and ...) in network results in an abridged version of the power network as follows:

$$
\dot{\overline{\mathbf{x}}}=\mathcal{A} \overline{\mathrm{x}}+\mathbf{w}
$$

where $\mathbf{w}$ represents the noise originating from modeling uncertainties, and $\overline{\mathbf{u}}$ is the input corresponding to reordered state vector with input matrix $\mathcal{B}$. For this simplified model, some parts of the power system may be entirely or partially unknown. In such cases, a cut is made to separate the known part from the unknown part. Then the states associated with unknown parts will be removed from the system dynamics. These unknown parts are modeled as unknown inputs flowing from the unknown part to the known part of the system. These unknown inputs are denoted by $d$, and the truncated state vector and its system matrix are represented by $\overline{\mathbf{x}}_{t r}$ and $\mathcal{A}_{t r}$, respectively. Thus, the model for a partially known power network can be given as:

$$
\dot{\overline{\mathbf{x}}}_{t r}=\mathcal{A}_{t r} \overline{\mathbf{x}}_{t r}+\mathbf{G d}+\mathbf{w}_{t r}
$$

where $\mathbf{w}_{t r}$ denotes the modeling noise for the partially known power network.

\section{E. The measurement model}

Modern PMUs can measure several variables in the power system, including bus voltage, bus current, valve position, and the output of the power system stabilizer [21], [22]. Moreover, state variables for a bus with a PMU can be measured directly [22]. In this case, we can choose the valve position $a_{k}$, the mechanical power $P_{m, k}$, rotor frequency $f_{r, k}$, and power angle $\delta_{k}$ as available measurements at generator $i$ with PMU [22]. Thus, the PMU-based measurement equation can be modeled as a linear function of states.

$$
\mathrm{y}=C \mathrm{x}+\mathrm{v}, \quad y, v \in \mathbb{R}^{p}
$$

This model fits the model (1)-(2), so the filtering algorithm described in Section II is applicable for partially known systems.

III. SENSOR PLACEMENT PROBLEM FORMULATION

This section aims to propose a systematic way for sensor placement for power network state estimation using S-SISE. The sensor selection vector is defined in [23] as

$$
\mathrm{w}=\left[w_{1}, \cdots, w_{m}\right]^{T}, \quad w_{k} \in\{0,1\},
$$

where $w_{k}$ specifies the availability of $i$ th sensor. Hence, the general measurement equation can be expressed as

$$
\begin{gathered}
y_{t, w}=C_{w} x_{t}+\Phi_{w} \nu_{t} \\
\text { and } C_{w}=\Phi_{w} C,
\end{gathered}
$$

where $\Phi_{w} \in\{0,1\}^{\|w\|_{1} p}$ is a matrix constructed from $\operatorname{diag}(\mathrm{w})$ such that all rows corresponding unselected sensors have been omitted from this matrix.

\section{A. Problem statement}

The problem we wish to solve can be stated as

$$
\min _{w} \operatorname{trace}\left(\mathcal{P}_{\infty, \mathrm{w}}\right) \quad \text { subject to } \quad 1^{T} \mathrm{w} \leq n_{s},
$$

with $\left(\mathcal{P}_{\infty, \mathrm{w}}\right)$ in (14) and defined above, where $n_{s} \leq p$ is the maximum number of sensors available. Note that Boolean variables in $w$ in the constraints of the optimization problem and the matrix $C_{w}$ the optimization problem make it nonconvex. The two lemmas below will be used in our first result, which shows that adding a sensor can only improve the cost in (19).

Lemma 2 ([24], [25]): For two selections w and $\tilde{\mathrm{w}}$, if $w_{k}=\tilde{w}_{k}$ for $i \in\{1, \cdots, m\} \backslash j, w_{j}=0$ and $\tilde{w}_{j}=1$ then $\mathcal{X}_{t+1, \tilde{\mathrm{w}}} \leq \mathcal{X}_{t+1, \mathrm{w}}$ and $\mathcal{X}_{\infty, \tilde{\mathrm{w}}} \leq \mathcal{X}_{\infty, \mathrm{w}}$.

Lemma 3 ([24], [26]): For two given matrices $A, B$ if $A \geq B$ then $A^{-1} \leq B^{-1}$

Let $C_{[k]}$ denote the $k$ th row of the measurement matrix $C$, and $R_{[k, k]}$ denote the $k$ th diagonal element of $R$. Next, we introduce the following definition:

Definition 1: Sensing precision matrix.

For each measurement $k$, the sensing precision matrix $S_{k}$, and its assimilated version $\tilde{S}$ are defined in [25], [27] as follows:

$$
\begin{array}{r}
S_{k}=C_{[k]}^{T} R_{[k, k]}^{-1} C_{[k]}, \\
\tilde{S}=\sum_{k=1}^{p} C_{[k]}^{T} R_{[k, k]}^{-1} C_{[k]}
\end{array}
$$


Remark 1: Note that the assimilated sensing precision matrix $\tilde{S}$ is the second term of the information matrix equation (10).

Theorem 1: If $\mathrm{w}$ and $\tilde{\mathrm{w}}$ are two sensor selections such that $w_{k}=\tilde{w}_{k}$ for $i \in\{1, \cdots, m\} \backslash j, w_{j}=0$ and $\tilde{w}_{j}=1$ then $\mathcal{P}_{\infty, \tilde{\mathrm{w}}} \leq \mathcal{P}_{\infty, \mathrm{w}}$.

Proof: Based on Lemma 3, we exploit information matrix for $t=0, \cdots, k-1$, and by using (10) and (20), we obtain

$$
\mathfrak{I}_{1, \tilde{\mathrm{w}}}=\mathcal{X}_{1, \tilde{\mathrm{w}}}^{-1}+\tilde{S}_{\mathrm{w}}+S_{j}
$$

where $\tilde{S}_{\mathrm{w}}$ is the assimilated sensing precision matrix for the sensor selection set w, and $S_{j}$ is the sensing precision matrix defined in (20). From Lemma 2 and Lemma 3, we have that $\mathcal{X}_{1, \tilde{\mathrm{w}}}^{-1} \geq \mathcal{X}_{1, \mathrm{w}}^{-1} ;$ thus

$$
\mathfrak{I}_{1, \tilde{\mathrm{w}}} \geq\left(\mathcal{X}_{1, \mathrm{w}}^{-1}+\tilde{S}_{\mathrm{w}}\right)+S_{j}=\mathfrak{I}_{1, \mathrm{w}}+S_{j}
$$

$S_{j} \geq 0$; hence,

$$
\mathfrak{I}_{1, \tilde{\mathrm{w}}} \geq \mathfrak{I}_{1, \mathrm{w}} .
$$

Repeating this procedure until $k-1$, yields

$$
\mathfrak{I}_{k, \tilde{\mathrm{w}}} \geq \mathfrak{I}_{k, \mathrm{w}} \cdot
$$

Then, following Lemma 1, we have

$$
\mathcal{P}_{k, \tilde{\mathrm{w}}} \leq \mathcal{P}_{k, \mathrm{w}},
$$

which holds for all $k$, letting $k \rightarrow \infty$ gives

$$
\mathcal{P}_{\infty, \tilde{\mathrm{w}}} \leq \mathcal{P}_{\infty, \mathrm{w}}
$$

As a result, adding a new sensor has no adverse effect on estimation accuracy. Therefore the inequality constraint in (19) can be changed to an equality constraint.

Note that, if we in KF-SISE also wish to take the input estimation into account, so the problem given in (19) can be extended to:

$$
\min _{w} \operatorname{trace}\left(\mathcal{P}_{\infty, \mathrm{w}}\right)+\operatorname{trace}\left(\mathcal{D}_{\infty, \mathrm{w}}\right) \quad \text { subject to } \quad 1^{T} \mathrm{w} \leq n_{s},
$$

Theorem 2: If $\mathrm{w}$ and $\tilde{\mathrm{w}}$ are two sensor selections such that $w_{k}=\tilde{w}_{k}$ for $i \in\{1, \cdots, m\} \backslash j, w_{j}=0$ and $\tilde{w}_{j}=1$ then $\mathcal{D}_{\tilde{\mathrm{w}}} \leq \mathcal{D}_{\mathrm{w}}$.

Proof: Take

$$
\mathcal{K}_{t+1}=\mathcal{X}_{t+1} C^{T}\left(C \mathcal{X}_{t+1} C^{T}+R\right)^{-1}
$$

By performing matrix inversion, we can obtain

$$
\mathcal{K}_{t+1}=\mathcal{P}_{t+1} C R^{-1}=\mathcal{X}_{t+1} C^{T}\left(C \mathcal{X} C^{T}+R\right)^{-1} .
$$

Thus,

$$
C^{T}\left(C \mathcal{X}_{t+1} C^{T}+R\right)^{-1}=\mathcal{X}_{t+1}^{-1} \mathcal{P}_{t+1} C R^{-1} .
$$

Therefore,

$$
\mathcal{M}_{t+1}=D G^{T} \mathcal{X}_{t+1}^{-1} \mathcal{P}_{t+1} C R^{-1}
$$

In addition,

$$
\mathcal{D}_{t}=\left(I-\mathcal{M}_{t+1} C G\right) D .
$$

Consider the second term of the above equation

$$
\mathcal{M}_{t+1} C G D=D G^{T} \mathcal{X}_{t+1}^{-1} \mathcal{P}_{t+1} C R^{-1} C G D .
$$

Now, we rewrite the above equation for $y_{t+1}^{\tilde{w}}$ as

$$
\mathcal{M}_{t+1}^{\tilde{w}} C_{\tilde{w}} G D=D G^{T} \mathcal{X}_{t+1}^{\tilde{w}}{ }^{-1} \mathcal{P}_{t+1}^{\tilde{w}} C_{\tilde{w}} R^{-1} C_{\tilde{w}} G D .
$$

Using Theorem 1 leads to:

$$
\begin{gathered}
\mathcal{M}_{t+1}^{\tilde{w}} C_{\tilde{w}} G D \\
\geq D G^{T} \mathcal{X}_{t+1}^{w}{ }^{-1}\left(\mathcal{X}_{t+1}^{w}{ }^{-1}+S_{j}\right)^{-1}\left(C_{w} R^{-1} C_{w}^{T}+S_{j}\right) G D .
\end{gathered}
$$

Note that since $\mathcal{P}_{t+1}^{w}{ }^{-1} \geq \mathcal{X}_{t+1}^{w}{ }^{-1} \geq C_{w}^{T} R^{-1} C_{w}$, we have

$$
\mathcal{M}_{t+1}^{\tilde{w}} C_{\tilde{w}} G D \geq D G^{T} \mathcal{X}_{t+1}^{w}{ }^{-1} \mathcal{P}_{t+1}^{w}\left(C_{w}^{T} R^{-1} C_{w}\right) G D,
$$

and then

$$
\mathcal{M}_{t+1}^{\tilde{w}} C_{\tilde{w}} G D \geq \mathcal{M}_{t+1}^{w} C_{w} G D
$$

which means

$$
\mathcal{D}_{t+1}^{\tilde{w}} \leq \mathcal{D}_{t+1}^{w}
$$

\section{B. Greedy algorithm for sensor placement}

This section proposes two methods using the greedy algorithm to address Problem (19). The solution is obtained such that the equality constraint in (19) holds. One algorithm starts with zero sensors, $n_{w}=0$, and increments $n_{w}$ until (19) holds; we call this the 'best in' greedy algorithm. The second algorithm starts with $n_{w}=p$ and decrease $n_{w}$ until $n_{w}=n_{s}$, we call this the greedy algorithm 'worst out'.

Note that Assumption 1. iii should be satisfied to reconstruct all signals of our interest, The two greedy algorithms are given in Algorithm 2 and 3. Algorithm 3 is called the

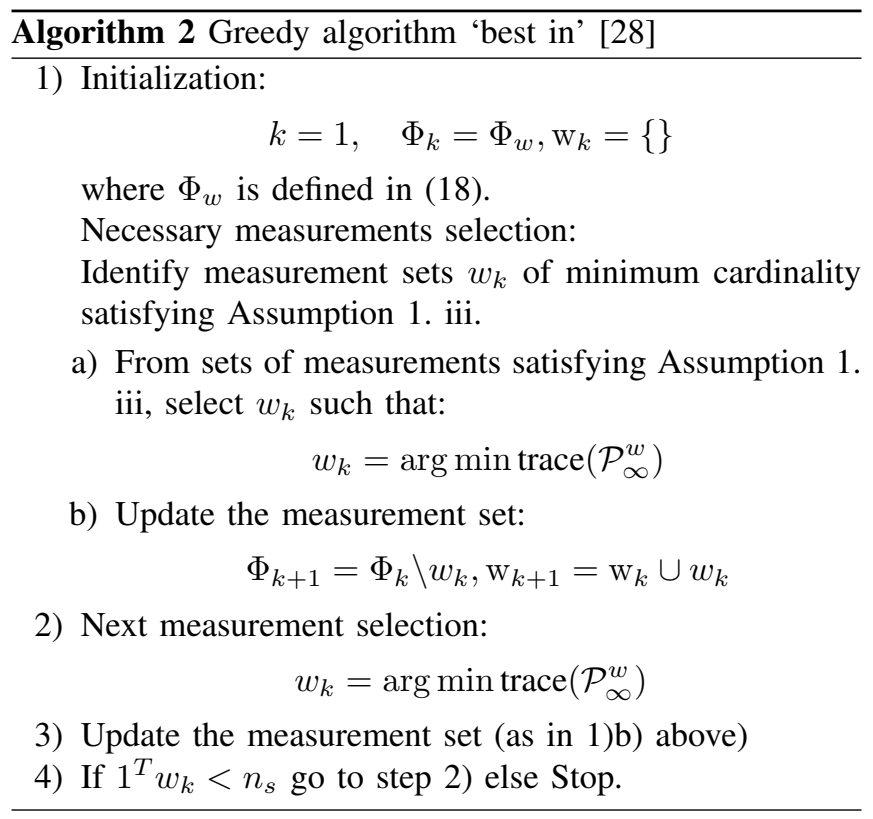

Greedy descent algorithm or greedy 'worst out' algorithm. In this algorithm, it is assumed that all measurements are available. Then the worst measurement from the measurement set would be omitted.

Remark 2: Algorithm 3 is easier to implement, and it does not need to perform a recoverability check (Assumption 1. iii). Hence, it is more straightforward to exploit Algorithm 3 when using the KF-SISE method in general. However, for large systems with a low number of sensors, Algorithm 2 might be used because of its lower computational cost and faster speed. 


\section{Algorithm 3 Greedy descent 'worst out'}

1) Initialization:

$$
k=1, \quad \Phi_{k}=\Phi_{w},
$$

and $\mathrm{w}_{k}$ is initialized as a vector of ones.

2) Next measurement selection for deletion:

$$
w_{k}=\arg \min \left|\operatorname{trace}\left(\mathcal{P}_{\infty}^{w}\left(\mathrm{w}_{k} \backslash\left\{w_{k}\right\}\right)\right)\right|
$$

3) Update the measurement set and select the subsequent measurement:

$$
\Phi_{k+1}=\Phi_{k} \backslash w_{k}, \mathrm{w}_{k+1}=\mathrm{w}_{k} \backslash w_{k}
$$

4) If $1^{T} w_{k}>n_{s}$ go to step 2) else Stop.

\section{NUMERICAL RESULTS}

In this part, we evaluate the proposed method's optimality using the WSCC-9 bus network Figure 1. To illustrate the proposed method, we assume each sensor can measure one state at each bus. Hence, we need to solve Problem (19) with $n_{s}=4$. After solving the Problem (19) with the Greedy algorithm given in Algorithm 2, we found that first, we need two measurements to assess the angular frequency at bus 1 and 2; in addition, measuring valve position first at bus one and then at bus two can give us the minimum value for our objective function, while Table II shows that this solution is the optimal solution for this problem.

We also implement Algorithm 3 for this problem. We start with the full set of available sensors (for simplicity, it is assumed the maximum number of sensors is nine and each sensor can measure only one state of the system), and then select the worst sensor concerning the optimality criteria and remove the worst sensor from the optimal sensor set. This is done until the optimal sensor set contains the maximum number of measurements that is $n_{s}=4$ here. Using Algorithm 3 yields removing $\omega_{r 4}, P_{1}, P_{2}, \delta_{2}, \delta_{4}$ from the optimal sensor set, respectively and results in $C_{\mathrm{w}^{*}}=$ $\left\{\omega_{r, 1}, \omega_{r, 2}, a_{1}, a_{2}\right\}$ as the optimal sensor set. This sensor set is the same as the one we obtained with Algorithm 2.

The estimation results for the WSCC-9-bus power system is given in Figure 2. We see that the estimates are accurate for the system states.

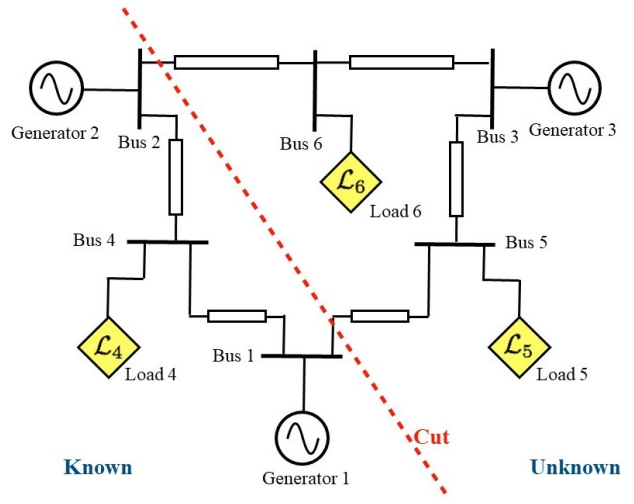

Fig. 1: WSCC-9 bus power system [8].

We further use the sensor selection method for the IEEE14 bus power system. Note that sensor placement strategies

\begin{tabular}{|l|l|}
\hline Measurements & trace $\mathcal{P}_{\infty}+$ trace $\mathcal{D}_{\infty}$ \\
\hline$\omega_{r 1}, \omega_{r 2}$ & $30.62+6.84$ \\
$\omega_{r 1}, \omega_{r 2}, \delta_{2}, \delta_{4}$ & $11.84+2.52$ \\
$\omega_{r 1}, \omega_{r 2}, \delta_{2}, a_{1}$ & $7.59+1.69$ \\
$\omega_{r 1}, \omega_{r 2}, \delta_{2}, P_{1}$ & $9.59+2.09$ \\
$\omega_{r 1}, \omega_{r 2}, \delta_{2}, a_{2}$ & $4.6+1.24$ \\
$\omega_{r 1}, \omega_{r 2}, \delta_{2}, P_{2}$ & $5.79+1.52$ \\
$\omega_{r 1}, \omega_{r 2}, \delta_{2}, \omega_{r 4}$ & $12.61+3.12$ \\
$\omega_{r 1}, \omega_{r 2}, \delta_{4}, a_{1}$ & $10.14+2.24$ \\
$\omega_{r 1}, \omega_{r 2}, \delta_{4}, P_{1}$ & $11.97+2.82$ \\
$\omega_{r 1}, \omega_{r 2}, \delta_{4}, a_{2}$ & $5.1+1.46$ \\
$\omega_{r 1}, \omega_{r 2}, \delta_{4}, P_{2}$ & $6.19+1.62$ \\
$\omega_{r 1}, \omega_{r 2}, \delta_{4}, \omega_{r 4}$ & $10.15+2.42$ \\
$\omega_{r 1}, \omega_{r 2}, a_{1}, P_{1}$ & $14.52+3.49$ \\
$\omega_{r 1}, \omega_{r 2}, a_{1}, a_{2}$ & $4.16+0.84$ \\
$\omega_{r 1}, \omega_{r 2}, a_{1}, P_{2}$ & $7.61+1.71$ \\
$\omega_{r 1}, \omega_{r 2}, a_{1}, \omega_{r 4}$ & $15.41+3.76$ \\
$\omega_{r 1}, \omega_{r 2}, P_{1}, a_{2}$ & $6.59+1.65$ \\
$\omega_{r 1}, \omega_{r 2}, P_{1}, P_{2}$ & $10.15+2.34$ \\
$\omega_{r 1}, \omega_{r 2}, P_{1}, \omega_{r 4}$ & $21.591+4.42$ \\
$\omega_{r 1}, \omega_{r 2}, a_{2}, P_{2}$ & $8.56+1.74$ \\
$\omega_{r 1}, \omega_{r 2}, a_{2}, \omega_{r 4}$ & $9.90+2.11$ \\
$\omega_{r 1}, \omega_{r 2}, P_{2}, \omega_{r} 4$ & $14.56+3.62$ \\
$\omega_{r 1}, \omega_{r 2}, P_{1}, P_{2}, \delta_{2}$ & $4.77+1.02$ \\
\hline \multicolumn{2}{|l}{}
\end{tabular}

TABLE II: Trace of $\mathcal{P}_{\infty}+$ trace of $\mathcal{D}_{\infty}$

based on steady-state/ quasi-steady-state conditions given in [15], [29], [11], [12] are not applicable here. The observability based sensor placement methods with different optimization criteria including steady-state error covariance [14], max, min and mean uncertainties [15], identifiability criteria [16], and the empirical observability Gramian [17] require at least five measurements to guarantee observability of the IEEE-14 bus power system with five generators and nine dynamic loads. From Table III, we can observe that by using the empirical observability Gramian method [17], and even five measurements, we cannot obtain accurate state estimates. However, by using the proposed method in this paper (Algorithm $2 \& 3$ ), the optimal sensor selection set is found as $\left\{\omega_{r, 1}, \omega_{r, 2}, \omega_{r, 3}\right\}$.

\begin{tabular}{lll}
\hline Placement methods & Measurements & min trace $\mathcal{P}_{\infty}$ \\
\hline Method in [17] & 5 & $7.34 \times 10^{6}$ \\
Algorithm 2 \& 3 & 3 & 86.75
\end{tabular}

TABLE III: Optimal sensor placement for the IEEE-14 bus power system

For this sensor selection, $\operatorname{trace}\left(\mathcal{P}_{\infty}+\mathcal{D}_{\infty}\right)=86.75+$ 17.61. Several state estimates and their actual states for IEEE-14 bus power system using KF-SISE and Algorithm $2 \& 3$ are depicted in Figure 4.

\section{CONCLUSion}

This paper presents two greedy algorithms for the placement of sensors in partially known power networks. Simulations are carried out on two power benchmarks, and results are presented. The information matrix for KF-SISE is derived, and it is utilized to address the optimization problem using the greedy algorithm. By considering the effects of disturbance on KF-SISE, we derive the new formulation for the optimization problem. This formulation accounts for both the state and input estimation error. The results are compared 

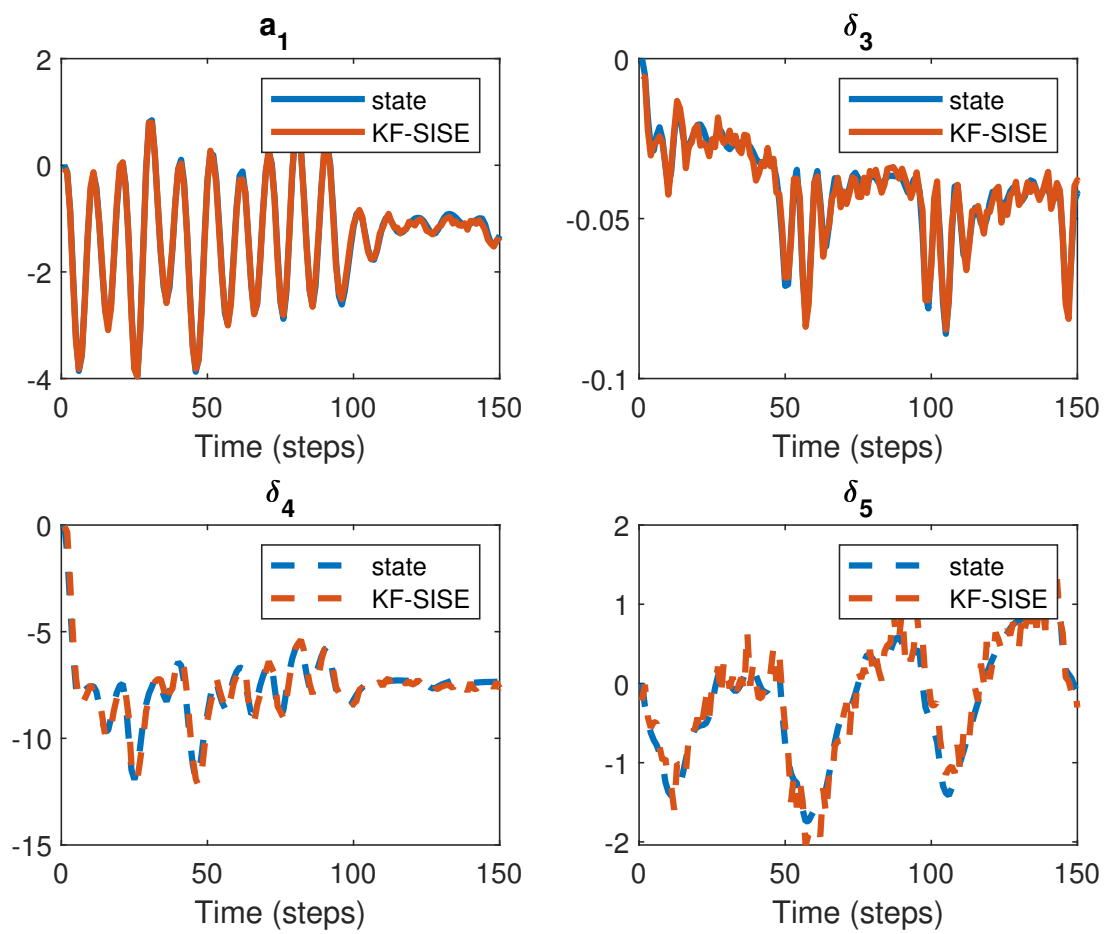

Fig. 2: State estimation for the WSCC-9-bus power system using KF-SISE and the proposed sensor placement algorithms.

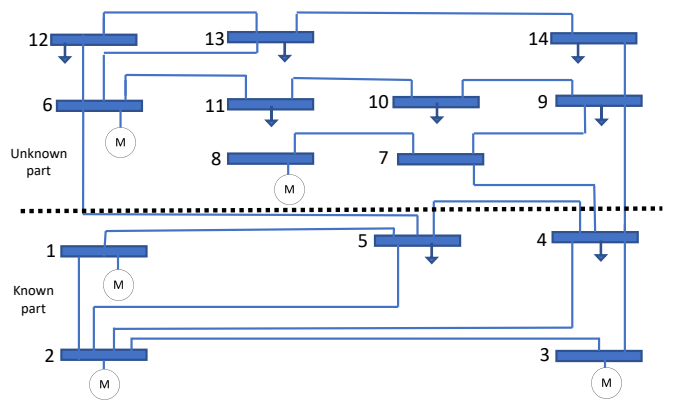

Fig. 3: Transformer-less dynamic power grid model of IEEE14-bus-system, with circuit cut dividing known and unknown parts [30].

to other sensor placement methods in order to validate the presented sensor placement method.

Future work is required for cases where measurement sets are not independent, and $R$ is not diagonal. We shall report on these problems elsewhere.

\section{REFERENCES}

[1] J. Zhao, A. Gómez-Expósito, M. Netto, L. Mili, A. Abur, V. Terzija, I. Kamwa, B. Pal, A. K. Singh, J. Qi, et al. Power system dynamic state estimation: Motivations, definitions, methodologies, and future work. IEEE Transactions on Power Systems, 34(4):3188-3198, 2019.

[2] M. Ghosal and V. Rao. Fusion of multirate measurements for nonlinear dynamic state estimation of the power systems. IEEE Transactions on Smart Grid, 10(1):216-226, Jan 2019.

[3] M. Jamei, A. Scaglione, C. Roberts, E. Stewart, S. Peisert, C. McParland, and A. McEachern. Anomaly detection using optimally placed $\mu \mathrm{PMU}$ sensors in distribution grids. IEEE Transactions on Power Systems, 33(4):3611-3623, 2018.

[4] T. Sadamoto, A. Chakrabortty, T. Ishizaki, and J. Imura. Dynamic modeling, stability, and control of power systems with distributed energy resources: Handling faults using two control methods in tandem. IEEE Control Systems Magazine, 39(2):34-65, April 2019.

[5] N. M. Manousakis, G. N. Korres, and P. S. Georgilakis. Taxonomy of PMU placement methodologies. IEEE Transactions on Power Systems, 27(2):1070-1077, 2012.

[6] V. Tran and H. Zhang. Optimal PMU placement using modified greedy algorithm. Journal of Control, Automation and Electrical Systems, 29(1):99-109, Feb 2018.

[7] Q. Li, T. Cui, Y. Weng, R. Negi, F. Franchetti, and M. D. Ilic. An information-theoretic approach to PMU placement in electric power systems. IEEE Transactions on Smart Grid, 4(1):446-456, 2012.

[8] M. A. Abooshahab, M. Hovd, and R. R. Bitmead. Disturbance and state estimation in partially known power networks. In 2019 IEEE Conference on Control Technology and Applications (CCTA), Aug 2019.

[9] R. R. Bitmead, M. Hovd, and M. A. Abooshahab. A Kalman-filtering derivation of simultaneous input and state estimation. Automatica, $108,2019$.

[10] M. A. Abooshahab, M. M.J. Alyaseen, R. R. Bitmead, and M. Hovd. Simultaneous input \& state estimation, singular filtering and stability (submitted for publication). arXiv preprint arXiv:2008.09217, 2020.

[11] L. Sun, T. Chen, X. Chen, W. K. Ho, K. Ling, K. Tseng, and G. A. J. Amaratunga. Optimum placement of phasor measurement units in power systems. IEEE Transactions on Instrumentation and Measurement, 68(2):421-429, 2019.

[12] N. Xie, F. Torelli, E. Bompard, and A. Vaccaro. A graph theory based methodology for optimal PMUs placement and multiarea power system state estimation. Electric Power Systems Research, 119:25-33, 2015.

[13] A. S. Zamzam and N. D. Sidiropoulos. Physics-aware neural networks for distribution system state estimation. IEEE Transactions on Power Systems, 35(6):4347-4356, 2020.

[14] P. Sun, Y.and Du, Z. Huang, K. Kalsi, R. Diao, K. K. Anderson, $\mathrm{Y}$. Li, and B. Lee. PMU placement for dynamic state tracking of power systems. In 2011 North American Power Symposium, pages $1-7,2011$.

[15] J. Zhang, G. Welch, and G. Bishop. Observability and estimation uncertainty analysis for PMU placement alternatives. In North American Power Symposium 2010, pages 1-8, 2010.

[16] P. Du, Z. Huang, R. Diao, B. Lee, and K. K. Anderson. PMU 

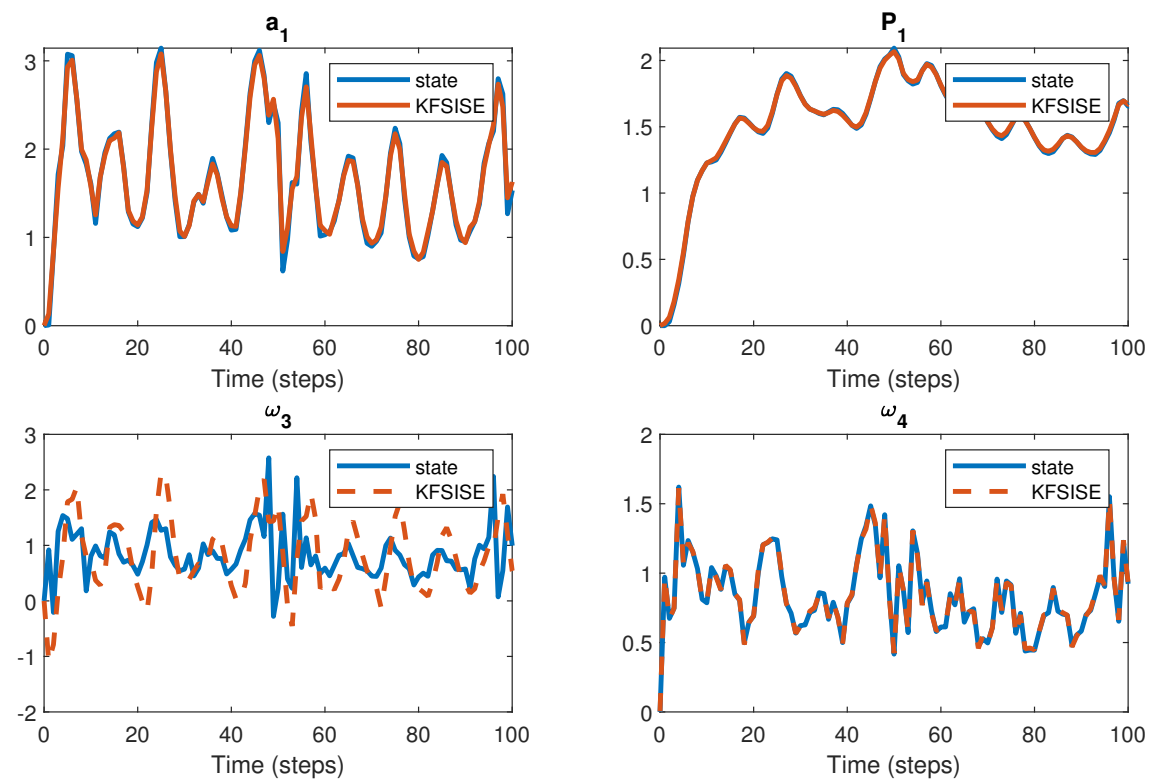

Fig. 4: State estimation for the IEEE-14-bus power system using KF-SISE and the proposed sensor placement algorithms.

placement for enhancing dynamic observability of a power grid. In 2010 IEEE Conference on Innovative Technologies for an Efficient and Reliable Electricity Supply, pages 15-21, 2010.

[17] J. Qi, K. Sun, and W. Kang. Optimal PMU placement for power system dynamic state estimation by using empirical observability gramian. IEEE Transactions on power Systems, 30(4):2041-2054, 2014.

[18] D. Van Hertem, J. Verboomen, K. Purchala, R. Belmans, and W. L. Kling. Usefulness of de power flow for active power flow analysis with flow controlling devices. In The 8th IEE International Conference on AC and DC Power Transmission, pages 58-62, March 2006.

[19] E. A. Blood. From Static to Dynamic Electric Power Network State Estimation: The Role of Bus Component Dynamics. PhD thesis, Carnegie Mellon University, 2011.

[20] P. Kundur, N. J. Balu, and M. G. Lauby. Power system stability and control, volume 7. McGraw-hill New York, 1994.

[21] Q. Yang, T. Bi, and J. Wu. WAMS implementation in China and the challenges for bulk power system protection. In 2007 IEEE Power Engineering Society General Meeting, pages 1-6, June 2007.

[22] S. Wang, W. Gao, and A. P. S. Meliopoulos. An alternative method for power system dynamic state estimation based on unscented transform. IEEE Transactions on Power Systems, 27(2):942-950, May 2012.

[23] S. Liu, S. P. Chepuri, M. Fardad, E. Maşazade, G. Leus, and P. K. Varshney. Sensor selection for estimation with correlated measurement noise. IEEE Transactions on Signal Processing, 64(13):3509-3522, 2016.

[24] H. Zhang, R. Ayoub, and S. Sundaram. Sensor selection for Kalman filtering of linear dynamical systems: Complexity, limitations and greedy algorithms. Automatica, 78:202 - 210, 2017.

[25] C. Yang, J. Wu, X. Ren, W. Yang, H. Shi, and L. Shi. Deterministic sensor selection for centralized state estimation under limited communication resource. IEEE Transactions on Signal Processing, 63(9):2336-2348, 2015.

[26] R. A. Horn and C. R. Johnson. Matrix analysis. Cambridge university press, 2012.

[27] C. Yang, J. Wu, W. Zhang, and L. Shi. Schedule communication for decentralized state estimation. IEEE Transactions on Signal Processing, 61(10):2525-2535, 2013.

[28] M. Shamaiah, S. Banerjee, and H. Vikalo. Greedy sensor selection: Leveraging submodularity. In 49th IEEE Conference on Decision and Control (CDC), pages 2572-2577, Dec 2010.

[29] V. Kekatos, G. B. Giannakis, and B. Wollenberg. Optimal placement of phasor measurement units via convex relaxation. IEEE Transactions on Power Systems, 27(3):1521-1530, 2012.

[30] M. A. Abooshahab, M. Hovd, and R. R. Bitmead. Kalman filter based monitoring of partially known power networks. Submitted to IEEE Transactions on Power Systems, 2020.

\section{APPENDIX}

Each unit (generator or load) at bus $k$ can be modeled as

$$
\dot{x}_{k}=A_{k} x_{k}+B_{k} u_{k}+B_{k}^{(G)} P_{G k} .
$$

where $B_{k}^{(G)}$ is the external input matrix corresponding to the external load. By aggregating the generator and load models, we can obtain

$$
\begin{aligned}
\mathbf{x} & =\left[x_{1}^{T}, \cdots, x_{k}^{T}, \cdots\right]^{T} \\
\mathbf{P} & =\left[P_{G 1}^{T}, \cdots, P_{G k}^{T}, \cdots\right]^{T} \\
\boldsymbol{\delta} & =\left[\delta_{1}^{T}, \cdots, \delta_{k}^{T}, \cdots\right]^{T} \\
\mathbf{A} & =\operatorname{blk}\left(A_{1}, \cdots, A_{k}, \cdots\right) \\
\mathbf{B}^{(\mathbf{G})} & =\operatorname{blk}\left(B_{1}^{(G)}, \cdots, B_{k}^{(G)}, \cdots\right) .
\end{aligned}
$$

where blk indicates a block diagonal matrix.

For the WSCC-9-bus power system, the reduced model susceptance matrix of the 9-bus network in Figure1 is given as follows:

$$
\mathrm{B}=\left[\begin{array}{cccccc}
-0.33 & 0 & 0 & 0.18 & 0.16 & 0 \\
0 & -0.45 & 0 & 0.31 & 0 & 0.15 \\
0 & 0 & -0.57 & 0 & 0.36 & 0.21 \\
0.18 & 0.31 & 0 & -0.48 & 0 & 0 \\
0.16 & 0 & 0.36 & 0 & -0.52 & 0 \\
0 & 0.15 & 0.21 & 0 & 0 & -0.36
\end{array}\right],
$$

Taking Assumption 1 into account, the approximations of the power signals are as follows:

$$
\mathbf{P}=\mathrm{B} \boldsymbol{\delta} .
$$

Now, we neglect the known inputs from the model description, so we obtain

$$
\dot{\mathbf{x}}=\mathbf{A} \mathbf{x}+\mathbf{B}^{(\mathbf{G})} \mathrm{B} \boldsymbol{\delta} .
$$

Furthermore,

$$
\dot{\mathbf{x}}=\left(\mathbf{A}+\mathbf{B}^{(\mathbf{G})} \mathrm{B} \Psi_{\delta}\right) \mathbf{x},
$$

where $\boldsymbol{\Psi}_{\delta}$ is a $\delta$ selection matrix $\boldsymbol{\delta}=\boldsymbol{\Psi}_{\delta} \mathbf{x}$. Without loss of generality, we reorder the state vector for each individual unit to simplify the observation of the interconnections in the system matrix.

$$
x_{k}=\left[\begin{array}{c}
\hat{x}_{k} \\
\delta_{k}
\end{array}\right], \quad k=1, \cdots, 6
$$

Likewise, we reorder the model matrices correspondingly:

$$
\begin{aligned}
A_{k}=\left[\begin{array}{r:r}
G_{k} & 0 \\
\hdashline 1 & 0
\end{array}\right], \quad k=1, \cdots, 3 \\
A_{k}=\left[\begin{array}{c:c}
L_{k} & 0 \\
\hdashline 1 & 0
\end{array}\right], \quad k=4, \cdots, 6
\end{aligned}
$$

where $G_{k}$ and $L_{k}$ are obtained as a result of this re-organization. For $k=1, \cdots, 6$, and the system state vector can be described as $\overline{\mathbf{x}}=$ $\left[\hat{x}_{1}^{T}, \cdots, \hat{x}_{k}^{T}, \cdots \delta_{2}^{T}, \cdots, \delta_{k}^{T}, \cdots\right]^{T}$. 